\title{
Coordinatewise decomposition, Borel cohomology, and invariant measures
}

\author{
by \\ Benjamin D. Miller (Los Angeles, CA)
}

\begin{abstract}
Given Polish spaces $X$ and $Y$ and a Borel set $S \subseteq X \times Y$ with countable sections, we describe the circumstances under which a Borel function $f: S \rightarrow \mathbb{R}$ is of the form $f(x, y)=u(x)+v(y)$, where $u: X \rightarrow \mathbb{R}$ and $v: Y \rightarrow \mathbb{R}$ are Borel. This turns out to be a special case of the problem of determining whether a real-valued Borel cocycle on a countable Borel equivalence relation is a coboundary. We use several Glimm-Effros style dichotomies to give a solution to this problem in terms of certain $\sigma$-finite measures on the underlying space. The main new technical ingredient is a characterization of the existence of type III measures of a given cocycle.
\end{abstract}

Suppose that $S \subseteq X \times Y$ and $G$ is a group. A coordinatewise decomposition of a function $f: S \rightarrow G$ is a pair $(u, v)$, where $u: X \rightarrow G, v: Y \rightarrow G$, and

$$
\forall(x, y) \in S(f(x, y)=u(x) v(y))
$$

If $X$ and $Y$ are Polish spaces, $G$ is a standard Borel group, and $u$ and $v$ are Borel, then we say that $(u, v)$ is a Borel coordinatewise decomposition of $f$. Our main goal here is to show that when $S$ is a Borel set with countable sections, $f: S \rightarrow G$ is Borel, and $G=\langle\mathbb{R},+\rangle$, the existence of a Borel coordinatewise decomposition can be characterized in terms of certain $\sigma$-finite measures on the disjoint union of $X$ and $Y$ (by a measure on a Polish space, we shall always mean a measure on its Borel subsets). Before getting to this, however, we first consider the existence of coordinatewise decompositions, without imposing any definability restrictions.

For the sake of notational convenience, we assume that $X \cap Y=\emptyset$. Associated with each set $S \subseteq X \times Y$ is the set $Z_{S}=X \cup Y$, the graph $\mathcal{G}_{S}=S \cup S^{-1}$ on $Z_{S}$, the equivalence relation $E_{S}$ on $Z_{S}$ whose equivalence classes are the connected components of $\mathcal{G}_{S}$, and the groupoid $\Gamma_{S}$ of all

2000 Mathematics Subject Classification: Primary 03E15; Secondary 28D05.

Key words and phrases: good sets, Glimm-Effros style dichotomies.

The author was supported in part by NSF VIGRE Grant DMS-0502315. 
paths through $\mathcal{G}_{S}$. We use $\gamma^{-1}$ to denote the reversal of a path $\gamma$, and $\gamma_{1} \gamma_{2}$ to denote the concatenation of paths $\gamma_{1}$ and $\gamma_{2}$. Observe that each function $f: S \rightarrow G$ extends to a unique groupoid homomorphism, i.e., there is a unique function $\varphi_{f}: \Gamma_{S} \rightarrow G$ such that:

(1) $\forall(x, y) \in S\left(\varphi_{f}(\langle x, y\rangle)=f(x, y)\right)$.

(2) $\forall \gamma \in \Gamma_{S}\left(\varphi_{f}\left(\gamma^{-1}\right)=\varphi_{f}(\gamma)^{-1}\right)$.

(3) $\forall \gamma, \gamma_{1}, \gamma_{2} \in \Gamma_{S}\left(\gamma=\gamma_{1} \gamma_{2} \Rightarrow \varphi_{f}(\gamma)=\varphi_{f}\left(\gamma_{1}\right) \varphi_{f}\left(\gamma_{2}\right)\right)$.

We say that $\gamma \in \Gamma_{S}$ is a loop if its initial and terminal points coincide. The following fact was proven essentially by Cowsik-Kłopotowski-Nadkarni [1]:

Proposition 1. Suppose that $X$ and $Y$ are disjoint, $S \subseteq X \times Y, G$ is a group, and $f: S \rightarrow G$. Then the following are equivalent:

(1) $f$ admits a coordinatewise decomposition.

(2) $\forall \gamma \in \Gamma_{S}\left(\gamma\right.$ is a loop $\left.\Rightarrow \varphi_{f}(\gamma)=1_{G}\right)$.

Proof. To see $(1) \Rightarrow(2)$, suppose that $(u, v)$ is a coordinatewise decomposition of $f$ and $\gamma$ is a loop. If $\gamma=\left\langle x_{0}, y_{0}, \ldots, x_{n}, y_{n}, x_{0}\right\rangle$, then

$$
\begin{aligned}
\varphi_{f}(\gamma) & =f\left(x_{0}, y_{0}\right) f\left(x_{1}, y_{0}\right)^{-1} \cdots f\left(x_{n}, y_{n}\right) f\left(x_{0}, y_{n}\right)^{-1} \\
& =\left(u\left(x_{0}\right) v\left(y_{0}\right)\right)\left(u\left(x_{1}\right) v\left(y_{0}\right)\right)^{-1} \cdots\left(u\left(x_{n}\right) v\left(y_{n}\right)\right)\left(u\left(x_{0}\right) v\left(y_{n}\right)\right)^{-1}=1_{G} .
\end{aligned}
$$

The case that $\gamma=\left\langle y_{0}, x_{0}, \ldots, y_{n}, x_{n}, y_{0}\right\rangle$ is handled similarly.

To see $(2) \Rightarrow(1)$, fix a transversal $B \subseteq Z_{S}$ of $E_{S}$ (i.e., a set which intersects every $E_{S}$-class in exactly one point), and let $d$ be the graph metric associated with $\mathcal{G}_{S}$. Fix $g: Z_{S} \backslash B \rightarrow Z_{S}$ such that

$$
\forall z \in Z_{S} \backslash B\left((z, g(z)) \in \mathcal{G}_{S} \text { and } d(g(z), B)<d(z, B)\right),
$$

and define recursively $u: X \rightarrow G$ and $v: Y \rightarrow G$ by

$$
\begin{aligned}
& u(x)= \begin{cases}1_{G} & \text { if } x \in B, \\
f(x, g(x)) v(g(x))^{-1} & \text { otherwise, }\end{cases} \\
& v(y)= \begin{cases}1_{G} & \text { if } y \in B, \\
u(g(y))^{-1} f(g(y), y) & \text { otherwise. }\end{cases}
\end{aligned}
$$

To see that $(u, v)$ is a coordinatewise decomposition of $f$, note first that if $g(x)=y$, then $u(x)=f(x, y) v(y)^{-1}$, thus $f(x, y)=u(x) v(y)$. Similarly, if $g(y)=x$, then $v(y)=u(x)^{-1} f(x, y)$, thus $f(x, y)=u(x) v(y)$.

Finally, suppose that $\left(x_{0}, y_{0}\right) \in S \backslash\left(\operatorname{graph}(g) \cup \operatorname{graph}\left(g^{-1}\right)\right)$, and fix a loop $\gamma=\left\langle x_{0}, y_{0}, \ldots, x_{n}, y_{n}, x_{0}\right\rangle$ such that, with the exception of $\left(x_{0}, y_{0}\right)$, successive pairs along $\gamma$ are in $\operatorname{graph}(g) \cup \operatorname{graph}\left(g^{-1}\right)$. Then $\gamma=\gamma_{1} \gamma_{2}$, where $\gamma_{1}=\left\langle x_{0}, y_{0}, x_{1}\right\rangle$ and $\gamma_{2}=\left\langle x_{1}, y_{1}, \ldots, x_{n}, y_{n}, x_{0}\right\rangle$. Observe now that

$$
\begin{aligned}
\varphi_{f}\left(\gamma_{2}\right) & =f\left(x_{1}, y_{1}\right) f\left(x_{2}, y_{1}\right)^{-1} \cdots f\left(x_{n}, y_{n}\right) f\left(x_{0}, y_{n}\right)^{-1} \\
& =u\left(x_{1}\right) v\left(y_{1}\right)\left(u\left(x_{2}\right) v\left(y_{1}\right)\right)^{-1} \cdots u\left(x_{n}\right) v\left(y_{n}\right)\left(u\left(x_{0}\right) v\left(y_{n}\right)\right)^{-1} \\
& =u\left(x_{1}\right) u\left(x_{0}\right)^{-1} .
\end{aligned}
$$


As $\varphi_{f}\left(\gamma_{1}\right) \varphi_{f}\left(\gamma_{2}\right)=\varphi_{f}(\gamma)=1$, it follows that $\varphi_{f}\left(\gamma_{1}\right)=\varphi_{f}\left(\gamma_{2}\right)^{-1}$, thus

$$
u\left(x_{0}\right) u\left(x_{1}\right)^{-1}=\varphi_{f}\left(\gamma_{1}\right)=f\left(x_{0}, y_{0}\right) f\left(x_{1}, y_{0}\right)^{-1}=f\left(x_{0}, y_{0}\right)\left(u\left(x_{1}\right) v\left(y_{0}\right)\right)^{-1} \text {, }
$$

and it easily follows that $f\left(x_{0}, y_{0}\right)=u\left(x_{0}\right) v\left(y_{0}\right)$.

We now turn back to our main question, which, in the special case that $G=\langle\mathbb{C},+\rangle$, was considered earlier by Cowsik-Kłopotowski-Nadkarni [1]:

Question 2. Suppose that $X$ and $Y$ are disjoint Polish spaces, $S \subseteq$ $X \times Y$ is Borel, $G$ is a standard Borel group, and $f: S \rightarrow G$ is Borel. Under what circumstances does $f$ admit a Borel coordinatewise decomposition?

Suppose that $X$ is a Polish space, $E$ is an equivalence relation on $X$, and $G$ is a standard Borel group. We say that $\varrho: E \rightarrow G$ is a cocycle if

$$
\forall x E y E z(\varrho(x, z)=\varrho(x, y) \varrho(y, z)) .
$$

We say that cocycles $\varrho_{1}, \varrho_{2}: E \rightarrow G$ are (Borel) cohomologous if there is a Borel function $w: X \rightarrow G$ such that $\forall x E y\left(\varrho_{1}(x, y)=w(x) \varrho_{2}(x, y) w(y)^{-1}\right)$, and a cocycle $\varrho: E \rightarrow G$ is a (Borel) coboundary if it is cohomologous to the trivial cocycle, i.e., if there is a Borel function $w: X \rightarrow G$ such that $\forall x E y\left(\varrho(x, y)=w(x) w(y)^{-1}\right)$. Note that if $G$ is abelian, then $\varrho_{1}, \varrho_{2}: E \rightarrow G$ are cohomologous if and only if $\varrho(x, y)=\varrho_{1}(x, y) \varrho_{2}(x, y)^{-1}$ is a coboundary.

As we have already answered the non-descriptive version of Question 2, let us assume that $f$ admits a coordinatewise decomposition. In this case, Proposition 1 ensures that if $\gamma_{1}, \gamma_{2} \in \Gamma_{S}$ have the same initial and terminal points, then $\varphi_{f}\left(\gamma_{1}\right)=\varphi_{f}\left(\gamma_{2}\right)$, so we can define $\varrho_{f}: E_{S} \rightarrow G$ by

$$
\varrho_{f}(x, y)=\varphi_{f}(\gamma)
$$

where $\gamma \in \Gamma_{S}$ is any path from $x$ to $y$. As $\varphi_{f}$ is a groupoid homomorphism, it follows that $\varrho_{f}$ is a cocycle. Note also that if $E_{S}$ is Borel (which holds, for example, if $S$ has countable sections), then so too is $\varrho_{f}$.

Proposition 3. Suppose that $X$ and $Y$ are disjoint Polish spaces, $S \subseteq$ $X \times Y$ is Borel, $G$ is a standard Borel group, and $f: S \rightarrow G$ is a Borel function that admits a coordinatewise decomposition. Then the following are equivalent:

(1) $f$ admits a Borel coordinatewise decomposition.

(2) $\varrho_{f}$ is a coboundary.

Proof. To see $(1) \Rightarrow(2)$, suppose that $(u, v)$ is a Borel coordinatewise decomposition of $f$, define $w: Z_{S} \rightarrow G$ by

$$
w(z)= \begin{cases}u(z) & \text { if } z \in X, \\ v(z)^{-1} & \text { if } z \in Y,\end{cases}
$$

and set $\Gamma=\left\{\left\langle z_{1}, \ldots, z_{n}\right\rangle \in \Gamma_{S}: \varphi_{f}\left(\left\langle z_{1}, \ldots, z_{n}\right\rangle\right)=w\left(z_{1}\right) w\left(z_{n}\right)^{-1}\right\}$. If 
$(x, y) \in S$, then

$$
\varphi_{f}(\langle x, y\rangle)=f(x, y)=u(x) v(y)=w(x) w(y)^{-1},
$$

thus $\langle x, y\rangle \in \Gamma$. As $\Gamma$ is closed under reversal and concatenation, it follows that $\Gamma=\Gamma_{S}$. As any two $E_{S}$-related points $z_{1}, z_{2} \in Z_{S}$ are connected by a path $\gamma \in \Gamma_{S}$ from $z_{1}$ to $z_{2}$, it follows that

$$
\varrho_{f}\left(z_{1}, z_{2}\right)=\varphi_{f}(\gamma)=w\left(z_{1}\right) w\left(z_{2}\right)^{-1}
$$

thus $\varrho_{f}$ is a coboundary.

To see $(2) \Rightarrow(1)$, suppose that $w: Z_{S} \rightarrow G$ is a Borel function such that

$$
\forall z_{1} E_{S} z_{2}\left(\varrho_{f}\left(z_{1}, z_{2}\right)=w\left(z_{1}\right) w\left(z_{2}\right)^{-1}\right),
$$

define $u: X \rightarrow G$ and $v: Y \rightarrow G$ by

$$
u(x)=w(x) \quad \text { and } \quad v(y)=w(y)^{-1},
$$

and note that for all $(x, y) \in S$,

$$
f(x, y)=\varphi_{f}(\langle x, y\rangle)=\varrho_{f}(x, y)=w(x) w(y)^{-1}=u(x) v(y),
$$

thus $(u, v)$ is a Borel coordinatewise decomposition of $f$.

Proposition 3 shows that Question 2 is a special case of:

QUESTION 4. Under what circumstances is a cocycle a coboundary?

We will answer the special case of Question 4 in which $E$ is a countable Borel equivalence relation, $\varrho: E \rightarrow G$ is Borel, and $G=\langle\mathbb{R},+\rangle$. This will, in turn, give also an answer to the special case of Question 2 in which $S$ has countable sections and $G=\langle\mathbb{R},+\rangle$. For notational convenience, we work with $\langle(0, \infty), \cdot\rangle$ instead of $\langle\mathbb{R},+\rangle$.

We begin by noting a simple measure-theoretic restriction imposed upon cohomologous Borel cocycles. We use $[E]$ to denote the group of all Borel automorphisms $f: X \rightarrow X$ such that $\operatorname{graph}(f) \subseteq E$. A measure $\mu$ on $X$ is $E$-invariant if every element of $[E]$ is $\mu$-preserving, and $\mu$ is $\varrho$-invariant if for every Borel function $\varphi: X \rightarrow(0, \infty)$ and $f \in[E]$, we have

$$
\int \varphi(x) d f_{*} \mu(x)=\int \varphi(x) \varrho\left(f^{-1}(x), x\right) d \mu(x) .
$$

When $\varrho$ is the trivial cocycle, this says exactly that $\mu$ is $E$-invariant.

Proposition 5. Suppose that $X$ is a Polish space, $E$ is a countable Borel equivalence relation on $X$, and $\varrho_{1}, \varrho_{2}: E \rightarrow(0, \infty)$ are cohomologous Borel cocycles. Then every $\varrho_{1}$-invariant, $\sigma$-finite measure is equivalent to a $\varrho_{2}$-invariant, $\sigma$-finite measure.

Proof. Suppose that $\mu_{1}$ is a $\varrho_{1}$-invariant, $\sigma$-finite measure, fix a Borel function $w: X \rightarrow(0, \infty)$ such that $\forall x E y\left(\varrho_{2}(x, y) / \varrho_{1}(x, y)=w(x) / w(y)\right)$, 
and set $\mu_{2}=\int w d \mu_{1}$. It is clear that $\mu_{1} \sim \mu_{2}$ and $\mu_{2}$ is $\sigma$-finite, and if $\varphi: X \rightarrow(0, \infty)$ is Borel and $f \in[E]$, then

$$
\begin{aligned}
\int \varphi(x) d f_{*} \mu_{2}(x) & =\int \varphi(f(x)) d \mu_{2}(x)=\int \varphi(f(x)) w(x) d \mu_{1}(x) \\
& =\int \varphi(x) w\left(f^{-1}(x)\right) d f_{*} \mu_{1}(x) \\
& =\int \varphi(x) w\left(f^{-1}(x)\right) \varrho_{1}\left(f^{-1}(x), x\right) d \mu_{1}(x) \\
& =\int \varphi(x) \varrho_{2}\left(f^{-1}(x), x\right) w(x) d \mu_{1}(x) \\
& =\int \varphi(x) \varrho_{2}\left(f^{-1}(x), x\right) d \mu_{2}(x),
\end{aligned}
$$

thus $\mu_{2}$ is $\varrho_{2}$-invariant.

In particular, we obtain the following:

Corollary 6. Suppose that $X$ is a Polish space, $E$ is a countable Borel equivalence relation on $X$, and $\varrho: E \rightarrow(0, \infty)$ is a Borel cocycle. If $\varrho$ is a coboundary, then for every $\sigma$-finite measure $\mu$ on $X$, the following are equivalent:

(1) There is a $\sigma$-finite, E-invariant measure equivalent to $\mu$.

(2) There is a $\sigma$-finite, $\varrho$-invariant measure equivalent to $\mu$.

The main result of this paper is that conversely, if conditions (1) and (2) of Corollary 6 are equivalent, then $\varrho$ is a coboundary. The proof consists essentially of chaining together 3 different Glimm-Effros style dichotomies, each of which characterizes the circumstances under which $E$ admits a $\sigma$ finite measure of a particular type, in terms of appropriate $\sigma$-ideals on the underlying space. We describe next these dichotomy theorems which, for the sake of clarity, we actually state as equivalences.

A set $A \subseteq X$ is a partial transversal of $E$ if it intersects every equivalence class of $E$ in at most one point. Let $\mathcal{I}_{\text {smooth }}$ denote the $\sigma$-ideal generated by the Borel partial transversals of $E$. Given $x \in X$, we use $[x]_{E}$ to denote the $E$-class of $x$, and we say that a set $A \subseteq X$ is $E$-invariant if for all $x \in A$, the set $[x]_{E}$ is contained in $A$. A measure $\mu$ on $X$ is $E$-ergodic if every $E$-invariant Borel set is $\mu$-null or $\mu$-conull. Shelah-Weiss [5] have shown essentially the following:

Theorem 7. Suppose that $X$ is a Polish space and $E$ is a countable Borel equivalence relation on $X$. Then the following are equivalent:

(1) $X \notin \mathcal{I}_{\text {smooth }}$.

(2) There is an atomless, E-ergodic, E-invariant, $\sigma$-finite measure.

A set $A \subseteq X$ is $\varrho$-discrete if there exists $\varepsilon>0$ such that

$\forall x, y \in A(x E y \Rightarrow(x=y$ or $\varrho(x, y) \leq 1 /(1+\varepsilon)$ or $\varrho(x, y) \geq 1+\varepsilon))$.

Let $\mathcal{I}_{\text {discrete }}$ denote the $\sigma$-ideal generated by the $\varrho$-discrete Borel sets. 
A measure $\mu$ is $E$-quasi-invariant if every $f \in[E]$ sends $\mu$-null sets to $\mu$-null sets. As noted in $\S 2$ of Miller [4], every $E$-quasi-invariant, $\sigma$-finite measure is invariant with respect to some Borel cocycle $\varrho: E \rightarrow(0, \infty)$, and moreover, this cocycle is unique modulo $E$-invariant null sets. The family of $E$-ergodic, $E$-quasi-invariant, $\sigma$-finite measures can be broken into three types. We say that $\mu$ is of type $I$ if it is atomic, $\mu$ is of type $I I$ if it is equivalent to an atomless, $E$-invariant, $E$-ergodic, $\sigma$-finite measure on $X$, and $\mu$ is of type III otherwise. The following fact was shown essentially in $\S 3$ of Miller [4]:

Theorem 8. Suppose that $X$ is a Polish space, $E$ is a countable Borel equivalence relation on $X$, and $\varrho: E \rightarrow(0, \infty)$ is a Borel cocycle. Then the following are equivalent:

(1) $X \notin \mathcal{I}_{\text {discrete }}$.

(2) There is a @-invariant measure of type II.

(3) There is a @-invariant measure of type II or III.

We will actually need only the easy direction of Theorem 8; the full result is stated above so as to present a more detailed picture of the interaction between the $\sigma$-ideal generated by the $\varrho$-discrete Borel sets and the set of measures on the underlying space.

A set $A \subseteq X$ is $\varrho$-bounded if there exists $\varepsilon>0$ such that

$$
\forall x, y \in A(x E y \Rightarrow 1 /(1+\varepsilon) \leq \varrho(x, y) \leq 1+\varepsilon) .
$$

Let $\mathcal{I}_{\text {bounded }}$ denote the $\sigma$-ideal generated by the $\varrho$-bounded Borel sets.

Proposition 9. Suppose that $X$ is a Polish space, $E$ is a countable Borel equivalence relation on $X$, and $\varrho: E \rightarrow(0, \infty)$ is a Borel cocycle. Then the following are equivalent:

(1) $X \in \mathcal{I}_{\text {bounded }}$.

(2) $\varrho$ is a coboundary.

Proof. To see $(1) \Rightarrow(2)$, suppose that $B_{0}, B_{1}, \ldots \subseteq X$ are $\varrho$-bounded Borel sets such that $X=\bigcup_{n \in \mathbb{N}} B_{n}$, associate with each $x \in X$ the least $n(x) \in \mathbb{N}$ such that $B_{n(x)} \cap[x]_{E} \neq \emptyset$, and define $w: X \rightarrow(0, \infty)$ by

$$
w(x)=\sup \left\{\varrho(x, z): z \in B_{n(x)} \cap[x]_{E}\right\} .
$$

Suppose now that $x, y$ lie in the same $E$-class $C$. Fix $\varepsilon>0$, choose $z \in C$ such that $w(x) \leq \varrho(x, z)(1+\varepsilon)$ and $w(y) \leq \varrho(y, z)(1+\varepsilon)$, and observe that

$$
\begin{aligned}
\varrho(x, z) / \varrho(y, z)(1+\varepsilon) & \leq w(x) / w(y) \\
& \leq \varrho(x, z)(1+\varepsilon) / \varrho(y, z) .
\end{aligned}
$$

As $\varrho(x, z) / \varrho(y, z)=\varrho(x, y)$ and $\varepsilon>0$ was arbitrary, it follows that $\varrho(x, y)=$ $w(x) / w(y)$, thus $\varrho$ is a coboundary. 
To see $(2) \Rightarrow(1)$, suppose that $w: X \rightarrow(0, \infty)$ is a Borel function such that $\varrho(x, y)=w(x) / w(y)$, and observe that the sets $w^{-1}([1 / n, n])$ for $n \in \mathbb{Z}^{+}$ are $\varrho$-bounded and cover $X$.

This leads to the last of our three dichotomies, which is also the only one that is new, and consequently, the only one that we shall prove here. We will state this dichotomy in terms of the $\sigma$-ideal

$$
\mathcal{I}_{\text {bounded }} \vee \mathcal{I}_{\text {discrete }}=\left\{A \cup B: A \in \mathcal{I}_{\text {bounded }} \text { and } B \in \mathcal{I}_{\text {discrete }}\right\} \text {. }
$$

Theorem 10. Suppose that $X$ is a Polish space, $E$ is a countable Borel equivalence relation on $X$, and $\varrho: E \rightarrow(0, \infty)$ is a Borel cocycle. Then the following are equivalent:

(1) $X \notin \mathcal{I}_{\text {bounded }} \vee \mathcal{I}_{\text {discrete }}$.

(2) There is a @-invariant measure of type III.

Proof. The E-saturation of a set $A \subseteq X$ is given by

$$
[A]_{E}=\{x \in X: \exists y \in A(x E y)\} .
$$

In $\S 3$ of Miller [4], it is shown that $\mathcal{I}_{\text {discrete }}$ is closed under $E$-saturation. While we could get away with just this, it seems worth noting the following:

LEMma 11. $\mathcal{I}_{\text {bounded }}$ is closed under E-saturation.

Proof. It is enough to show that the $E$-saturation of every $\varrho$-bounded Borel set is in $\mathcal{I}_{\text {bounded }}$. Towards this end, suppose that $A \subseteq X$ is a $\varrho$-bounded Borel set, and note that the sets

$$
A_{n}=\{x \in X: \exists y \in A(1 / n \leq \varrho(y, x) \leq n)\}
$$

are $\varrho$-bounded and cover $[A]_{E}$, thus $[A]_{E} \in \mathcal{I}_{\text {bounded }}$.

To see $\neg(1) \Rightarrow \neg(2)$ of Theorem 10 , suppose that $X \in \mathcal{I}_{\text {bounded }} \vee \mathcal{I}_{\text {discrete }}$, and note that Lemma 11 ensures the existence of an $E$-invariant Borel set $B \in \mathcal{I}_{\text {discrete }}$ such that $X \backslash B \in \mathcal{I}_{\text {bounded }}$. Theorem 8 ensures that there are no $\varrho \mid B$-invariant measures of types II or III, and Corollary 6 and Proposition 9 ensure that there are no $\varrho \mid(X \backslash B)$-invariant measures of type III.

It remains to show $(1) \Rightarrow(2)$. Roughly speaking, we will produce an embedding of a specific sort of cocycle into $\varrho$, and then push an appropriate measure through this embedding in order to obtain the measure we desire. To better motivate the sort of embedding we will produce, we describe first a family of measures of type III which contains the measure that we shall push forward.

For $k \in \mathbb{Z}^{+}$, let $\mu_{k}$ be the probability measure on $\{0, \ldots, k\}$ given by

$$
\mu_{k}(\{i\})= \begin{cases}1 / 2 & \text { if } i=0, \\ 1 / 2 k & \text { otherwise. }\end{cases}
$$


For $\mathbf{k}=\left\langle k_{n}\right\rangle_{n \in \mathbb{N}}$ in $\left(\mathbb{Z}^{+}\right)^{\mathbb{N}}$, set $X_{\mathbf{k}}=\prod_{n \in \mathbb{N}}\left\{0, \ldots, k_{n}\right\}$, define $\mu_{\mathbf{k}}$ on $X_{\mathbf{k}}$ by $\mu_{\mathbf{k}}=\prod_{n \in \mathbb{N}} \mu_{k_{n}}$, and define $E_{\mathbf{k}}$ on $X_{\mathbf{k}}$ by

$$
\alpha E_{\mathbf{k}} \beta \Leftrightarrow \exists n \in \mathbb{N} \forall m \geq n(\alpha(m)=\beta(m)) .
$$

Set $\varrho_{k}(i, j)=\mu_{k}(\{i\}) / \mu_{k}(\{j\})$, and define $\varrho_{\mathbf{k}}: E_{\mathbf{k}} \rightarrow(0, \infty)$ by

$$
\varrho_{\mathbf{k}}(\alpha, \beta)=\prod_{n \in \mathbb{N}} \varrho_{k_{n}}(\alpha(n), \beta(n)) .
$$

It follows from Proposition 2.4 of Miller [4] that $\mu_{\mathbf{k}}$ is $\varrho_{\mathbf{k}}$-invariant.

Lemma 12. If $\lim \sup _{n \rightarrow \infty} k_{n}=\infty$, then $\left(X_{\mathbf{k}}, E_{\mathbf{k}}, \mu_{\mathbf{k}}\right)$ is of type III.

Proof. It is clear that $\mu_{\mathbf{k}}$ is atomless, and it follows from the analog of the Lebesgue density theorem in $X_{\mathbf{k}}$ (see $\S 2$ of Miller [4]) that $\mu_{\mathbf{k}}$ is $E_{\mathbf{k}}$-ergodic. Suppose, towards a contradiction, that there is an $E_{\mathbf{k}}$-invariant, $\sigma$-finite measure $\mu \sim \mu_{\mathbf{k}}$. Fix a Borel function $w: X_{\mathbf{k}} \rightarrow(0, \infty)$ such that $\mu_{\mathbf{k}}=\int w d \mu$, and note that if $\varphi: X \rightarrow(0, \infty)$ is Borel and $f \in\left[E_{\mathbf{k}}\right]$, then

$$
\begin{aligned}
\int \varphi(\alpha) d f_{*} \mu_{\mathbf{k}}(\alpha) & =\int \varphi(f(\alpha)) d \mu_{\mathbf{k}}(\alpha)=\int \varphi(f(\alpha)) w(\alpha) d \mu(\alpha) \\
& =\int \varphi(f(\alpha))(w(\alpha) / w(f(\alpha))) w(f(\alpha)) d \mu(\alpha) \\
& =\int \varphi(\alpha)\left(w\left(f^{-1}(\alpha)\right) / w(\alpha)\right) w(\alpha) d \mu(\alpha) \\
& =\int \varphi(\alpha)\left(w\left(f^{-1}(\alpha)\right) / w(\alpha)\right) d \mu_{\mathbf{k}}(\alpha) .
\end{aligned}
$$

We can therefore assume that $\varrho_{\mathbf{k}}(\alpha, \beta)=w(\alpha) / w(\beta)$.

Fix $0<\varepsilon<1$ sufficiently small that the set $B=w^{-1}([\varepsilon, 1 / \varepsilon])$ is of $\mu_{\mathbf{k}}$-measure strictly greater than $1 / 2$. Fix $n \in \mathbb{N}$ such that $k_{n}>1 / \varepsilon^{2}$, and for each $i \leq k_{n}$, define $f_{i} \in\left[E_{\mathbf{k}}\right]$ by

$$
\left[f_{i}(\alpha)\right](j)= \begin{cases}0 & \text { if } j=n \text { and } \alpha(n)=i, \\ i & \text { if } j=n \text { and } \alpha(n)=0, \\ \alpha(j) & \text { otherwise. }\end{cases}
$$

Let $A=\left\{\alpha \in X_{\mathbf{k}}: \alpha(n)=0\right\}$, and note that if $\alpha \in A$ and $i \in\left\{1, \ldots, k_{n}\right\}$, then $\varrho_{\mathbf{k}}\left(\alpha, f_{i}(\alpha)\right)=\varrho_{k_{n}}(0, i)=k_{n}>1 / \varepsilon^{2}$. In particular, if $\alpha \in A \cap B$, then none of $f_{1}(\alpha), \ldots, f_{k_{n}}(\alpha)$ are in $B$. This, in turn, implies that

$$
\sum_{i \leq k_{n}} \chi_{B}\left(f_{i}(\alpha)\right) \varrho_{\mathbf{k}}\left(f_{i}(\alpha), \alpha\right) \leq \frac{1}{2} \sum_{i \leq k_{n}} \varrho_{\mathbf{k}}\left(f_{i}(\alpha), \alpha\right)
$$

for all $\alpha \in A$. It now follows that

$$
\mu_{\mathbf{k}}(B)=\sum_{i \leq k_{n}} \mu_{\mathbf{k}}\left(f_{i}(A) \cap B\right)=\sum_{i \leq k_{n}} \mu_{\mathbf{k}}\left(f_{i}\left(A \cap f_{i}^{-1}(B)\right)\right)
$$




$$
\begin{aligned}
& =\sum_{i \leq k_{n}} \int_{A \cap f_{i}^{-1}(B)} \varrho_{\mathbf{k}}\left(f_{i}(\alpha), \alpha\right) d \mu_{\mathbf{k}}(\alpha) \\
& =\int_{A} \sum_{i \leq k_{n}} \chi_{B}\left(f_{i}(\alpha)\right) \varrho_{\mathbf{k}}\left(f_{i}(\alpha), \alpha\right) d \mu_{\mathbf{k}}(\alpha) \\
& \leq \frac{1}{2} \int_{A} \sum_{i \leq k_{n}} \varrho_{\mathbf{k}}\left(f_{i}(\alpha), \alpha\right) d \mu_{\mathbf{k}}(\alpha)=\frac{1}{2} \sum_{i \leq k_{n}} \mu_{\mathbf{k}}\left(f_{i}(A)\right)=\frac{1}{2},
\end{aligned}
$$

which is the desired contradiction.

An $\varepsilon$-embedding of $\varrho_{\mathbf{k}}$ into $\varrho$ is an embedding $\pi: X_{\mathbf{k}} \rightarrow X$ of $E_{\mathbf{k}}$ into $E$ such that $\forall \alpha E_{\mathbf{k}} \beta\left(\varrho_{\mathbf{k}}(\alpha, \beta) /(1+\varepsilon) \leq \varrho(\pi(\alpha), \pi(\beta)) \leq \varrho_{\mathbf{k}}(\alpha, \beta)(1+\varepsilon)\right)$. We will complete the proof of Theorem 10 by showing first the following descriptive strengthening:

Theorem 13. Suppose that $X$ is a Polish space, $E$ is a countable Borel equivalence relation on $X, \varrho: E \rightarrow(0, \infty)$ is a Borel cocycle, and $\varepsilon>0$. Then the following are equivalent:

(1) $X \notin \mathcal{I}_{\text {bounded }} \vee \mathcal{I}_{\text {discrete }}$.

(2) There is a continuous $\varepsilon$-embedding of $\varrho_{\mathbf{k}}$ into $\varrho$ for some $\mathbf{k}=\left\langle k_{n}\right\rangle_{n \in \mathbb{N}}$ such that $\lim _{n \rightarrow \infty} k_{n}=\infty$.

Proof. In order to see that $\neg(1) \Rightarrow \neg(2)$ suppose, towards a contradiction, that both $\neg(1)$ and (2) hold. Note that pre-images under $\varepsilon$-embeddings preserve the bounded and discrete $\sigma$-ideals, so that the join of the bounded and discrete $\sigma$-ideals corresponding to $\varrho_{\mathbf{k}}$ trivializes. However, Lemma 12 implies that $\mu_{\mathbf{k}}$ is a $\varrho_{\mathbf{k}}$-invariant measure of type III, thus $(2) \Rightarrow(1)$ of Theorem 10 implies that the the join of the bounded and discrete $\sigma$-ideals corresponding to $\varrho_{\mathbf{k}}$ does not trivialize, which is the desired contradiction.

It remains to show $(1) \Rightarrow(2)$. By Theorem 1 of Feldman-Moore [2], there is a countable group $\Gamma \leq[E]$ such that $E=E_{\Gamma}^{X}$. By change of topology results (see, for example, $\S 13$ of Kechris [3]), there is a finer zero-dimensional Polish topology $\tau$, compatible with the underlying Borel structure of $X$, with respect to which $\Gamma$ acts by homeomorphisms and each of the sets $\{x \in X$ : $k \leq \varrho(x, \gamma \cdot x)<r\}$ is open, where $\gamma \in \Gamma, k \in \mathbb{Z}^{+}$, and $r \in(k, \infty)$. Fix $\varepsilon_{n}>0$, for $n \in \mathbb{N}$, such that

$$
\prod_{n \in \mathbb{N}}\left(1+\varepsilon_{n}\right) \leq 1+\varepsilon,
$$

as well as finite, symmetric sets $\left\{1_{\Gamma}\right\}=\Gamma_{0} \subseteq \Gamma_{1} \subseteq \cdots \subseteq \Gamma$ such that $\Gamma=\bigcup_{n \in \mathbb{N}} \Gamma_{n}$. It will be convenient to set $\mathcal{I}=\mathcal{I}_{\text {bounded }} \vee \overline{\mathcal{I}}_{\text {discrete }}$ for the remainder of the proof.

We will recursively find $\tau$-clopen sets $B_{n} \subseteq X, k_{n} \in \mathbb{Z}^{+}$, and $\gamma_{n, k} \in \Gamma$, for $n \in \mathbb{N}$ and $k \leq k_{n}$. Associated with these are the sets $X_{n}=\prod_{i<n}\left\{0, \ldots, k_{i}\right\}$, 
the group elements $\gamma_{s}=\prod_{i<n} \gamma_{i, s(i)}$ for $s \in X_{n}$, and the sets

$$
\Delta_{n}=\left\{\gamma_{s}^{-1} \gamma \gamma_{t}: \gamma \in \Gamma_{n} \text { and } s, t \in X_{n}\right\} .
$$

We will ensure that, for all $n \in \mathbb{N}$, the following conditions are satisfied:

(1) $B_{n} \notin \mathcal{I}$.

(2) $k_{n} \geq n$.

(3) $\gamma_{n, 0}=1_{\Gamma}$.

(4) $\forall x \in B_{n+1} \forall k \leq k_{n}\left(\varrho_{k_{n}}(0, k) \leq \varrho\left(x, \gamma_{n, k} \cdot x\right)<\varrho_{k_{n}}(0, k)\left(1+\varepsilon_{n}\right)\right)$.

(5) $\forall s \in X_{n+1}\left(\operatorname{diam}\left(\gamma_{s}\left(B_{n+1}\right)\right) \leq 1 / n\right)$.

(6) $\forall j<k \leq k_{n}\left(\Delta_{n} \gamma_{n, j}\left(B_{n+1}\right) \cap \gamma_{n, k}\left(B_{n+1}\right)=\emptyset\right)$.

(7) $\forall k \leq k_{n}\left(\gamma_{n, k}\left(B_{n+1}\right) \subseteq B_{n}\right)$.

We begin by setting $B_{0}=X$. Suppose now that we have $B_{0} \supseteq B_{1} \supseteq \cdots$ $\cdots \supseteq B_{n}$, as well as $k_{i}$ and $\gamma_{i, k}$ for $k \leq k_{i}$ and $i<n$. Set $C_{0}=B_{n}$.

Lemma 14. There is an $\mathcal{I}$-positive, $\tau$-open set $C_{1} \subseteq C_{0}, \gamma_{n, 1} \in \Gamma$, and $k_{n} \geq n$ such that, for all $x \in C_{1}$, the following conditions are satisfied:

(a) $\gamma_{n, 1} \cdot x \in C_{0} \backslash \Delta_{n} \cdot x$.

(b) $\varrho_{k_{n}}(0,1) \leq \varrho\left(x, \gamma_{n, 1} \cdot x\right)<\varrho_{k_{n}}(0,1)\left(1+\varepsilon_{n}\right)$.

Proof. For each $\gamma \in \Gamma$ and $k \geq \max \left(n, 1 / \varepsilon_{n}\right)$, define $C_{\gamma, k} \subseteq C_{0}$ by $C_{\gamma, k}=\left\{x \in C_{0}: \gamma \cdot x \in C_{0} \backslash \Delta_{n} \cdot x\right.$ and $\left.k \leq \varrho(x, \gamma \cdot x)<k+1\right\}$, and set $C=C_{0} \backslash \bigcup\left\{C_{\gamma, k}: \gamma \in \Gamma\right.$ and $\left.k \geq \max \left(n, 1 / \varepsilon_{n}\right)\right\}$.

Sublemma 15. $C \in \mathcal{I}_{\text {bounded }}$.

Proof. Define $w: C \rightarrow[1, \infty]$ by

$$
w(x)=\sup \left\{\varrho(x, y): y \in C \cap[x]_{E}\right\},
$$

and given $x \in C$, note that if $y \in C \cap[x]_{E}$ and $\varrho(x, y) \geq \max \left(n, 1 / \varepsilon_{n}\right)+1$, then $y \in \Delta_{n} \cdot x$. In particular, it follows that $\forall x \in C(w(x)<\infty)$, thus $C=\bigcup_{n \in \mathbb{Z}^{+}} w^{-1}([1, n])$. As each of the sets $w^{-1}([1, n])$ is $\varrho$-bounded, it follows that $C \in \mathcal{I}_{\text {bounded. }}$.

Consequently, there exist $\gamma \in \Gamma$ and $k \geq \max \left(n, 1 / \varepsilon_{n}\right)$ such that $C_{\gamma, k} \notin \mathcal{I}$. Put $C_{1}=C_{\gamma, k}, \gamma_{n, 1}=\gamma$, and $k_{n}=k$, and note that $\varrho_{k_{n}}(0,1)=k_{n}$ and

$$
\varrho_{k_{n}}(0,1)\left(1+\varepsilon_{n}\right) \geq k_{n}\left(1+1 / k_{n}\right)=k_{n}+1,
$$

thus $\forall x \in C_{1}\left(\varrho_{k_{n}}(0,1) \leq \varrho\left(x, \gamma_{n, 1} \cdot x\right)<\varrho_{k_{n}}(0,1)\left(1+\varepsilon_{n}\right)\right)$.

Suppose now that $1 \leq k<k_{n}$ and we have found $\mathcal{I}$-positive, $\tau$-open sets $C_{0} \supseteq C_{1} \supseteq \cdots \supseteq C_{k}$ and $\gamma_{n, 0}, \gamma_{n, 1}, \ldots, \gamma_{n, k} \in \Gamma$. Set

$$
\Delta_{n, k}=\left\{\delta \gamma_{n, i}: \delta \in \Delta_{n} \text { and } i \leq k\right\} .
$$

Lemma 16. There is an $\mathcal{I}$-positive, $\tau$-open set $C_{k+1} \subseteq C_{k}$ and $\gamma_{n, k+1} \in \Gamma$ such that, for all $x \in C_{k+1}$, the following conditions are satisfied: 
(a) $\gamma_{n, k+1} \cdot x \in \gamma_{n, k}\left(C_{k}\right) \backslash \Delta_{n, k} \cdot x$.

(b) $\varrho_{k_{n}}(0, k+1) \leq \varrho\left(x, \gamma_{n, k+1} \cdot x\right)<\varrho_{k_{n}}(0, k+1)\left(1+\varepsilon_{n}\right)$.

Proof. For each $\gamma \in \Gamma$, let $D_{\gamma}$ be the set of $x \in \gamma_{n, k}\left(C_{k}\right)$ such that $\gamma \gamma_{n, k}^{-1} \cdot x \in \gamma_{n, k}\left(C_{k}\right) \backslash \Delta_{n, k} \gamma_{n, k}^{-1} \cdot x$ and $k_{n} \leq \varrho\left(\gamma_{n, k}^{-1} \cdot x, \gamma \gamma_{n, k}^{-1} \cdot x\right)<k_{n}\left(1+\varepsilon_{n}\right)$, and set $D=\gamma_{n, k}\left(C_{k}\right) \backslash \bigcup_{\gamma \in \Gamma} D_{\gamma}$.

Sublemma 17. $D \in \mathcal{I}_{\text {discrete }}$.

Proof. Define $F \subseteq E$ by

$$
x F y \Leftrightarrow(x E y \text { and } \varrho(x, y)=1) .
$$

Given $x \in D$, note that $k_{n} \leq \varrho\left(\gamma_{n, k}^{-1} \cdot x, x\right)<k_{n}\left(1+\varepsilon_{n}\right)$, so there exists $\delta>0$ such that if $y \in D \cap[x]_{E}$ and $1 \leq \varrho(x, y)<1+\delta$, then $y \in \Delta_{n, k} \gamma_{n, k}^{-1} \cdot x$. In particular, it follows that every equivalence class of $F \mid D$ is of cardinality at most $\left|\Delta_{n, k}\right|$, hence there are Borel partial transversals $D_{i}^{\prime}$ of $F$, for $i<\left|\Delta_{n, k}\right|$, whose union is $D$. For each $i<\left|\Delta_{n, k}\right|$ and $j \in \mathbb{N}$, let $D_{i, j}^{\prime}$ be the set of all $x \in D_{i}^{\prime}$ such that

$$
\forall y \in D_{i}^{\prime} \cap[x]_{E}(x=y \text { or } \varrho(x, y) \geq 1+1 / j \text { or } \varrho(y, x) \geq 1+1 / j) .
$$

These are clearly $\varrho$-discrete Borel sets which cover $D$, thus $D \in \mathcal{I}_{\text {discrete }}$.

It now follows that there exists $\gamma \in \Gamma$ such that the set $D_{\gamma}$ is $\mathcal{I}$-positive. Put $C_{k+1}=\gamma_{n, k}^{-1}\left(D_{\gamma}\right)$ and $\gamma_{n, k+1}=\gamma$, and observe that $\varrho_{k_{n}}(0, k+1)=k_{n}$, thus $\forall x \in C_{k+1}\left(\varrho_{k_{n}}(0, k+1) \leq \varrho\left(x, \gamma_{n, k+1} \cdot x\right)<\varrho_{k_{n}}(0, k+1)\left(1+\varepsilon_{n}\right)\right)$.

This completes the description of $C_{0}, C_{1}, \ldots, C_{k_{n}}$ and $\gamma_{n, 0}, \gamma_{n, 1}, \ldots, \gamma_{n, k_{n}}$. As $C_{k_{n}}$ is the union of countably many $\tau$-clopen sets $D \subseteq C_{k_{n}}$ which satisfy the analogs of conditions (5) and (6) in which $B_{n+1}$ is replaced with $D$, it follows that there is an $\mathcal{I}$-positive, $\tau$-clopen set $B_{n+1} \subseteq C_{k_{n}}$ which satisfies conditions (1)-(7).

This completes the recursive construction. For each $s \in X_{n}$, set $A_{s}=$ $\gamma_{s}\left(B_{n}\right)$. Put $\mathbf{k}=\left\langle k_{n}\right\rangle_{n \in \mathbb{N}}$, and note that for each $\alpha \in X_{\mathbf{k}}$, conditions (5) and (7) ensure that $A_{\alpha(0)}, A_{\alpha(0) \alpha(1)}, \ldots$ is a decreasing sequence of clopen sets with vanishing diameter. It follows that their intersection consists of a single point. Let $\pi(\alpha)$ denote this point. By conditions (5) and (6), the function $\pi: X_{\mathbf{k}} \rightarrow X$ is a continuous injection.

To see $\alpha E_{\mathbf{k}} \beta \Rightarrow \pi(\alpha) E \pi(\beta)$, it is enough to observe the following:

LEMmA 18. If $n \in \mathbb{N}, s \in X_{n}$, and $s \alpha \in X_{\mathbf{k}}$, then $\pi(s \alpha)=\gamma_{s} \cdot \pi\left(0^{n} \alpha\right)$. 
Proof. Simply observe that

$$
\begin{aligned}
\{\pi(s \alpha)\} & =\bigcap_{i \geq n} A_{(s \alpha) \mid i}=\bigcap_{i \in \mathbb{N}} \gamma_{s} \gamma_{0^{n}(\alpha \mid i)}\left(B_{i+n}\right)=\gamma_{s}\left(\bigcap_{i \in \mathbb{N}} \gamma_{0^{n}(\alpha \mid i)}\left(B_{i+n}\right)\right) \\
& =\gamma_{s}\left(\bigcap_{i \geq n} A_{0^{n}(\alpha \mid i)}\right)=\left\{\gamma_{s} \cdot \pi\left(0^{n} \alpha\right)\right\},
\end{aligned}
$$

thus $\pi(s \alpha)=\gamma_{s} \cdot \pi\left(0^{n} \alpha\right)$.

To see $(\alpha, \beta) \notin E_{\mathbf{k}} \Rightarrow(\pi(\alpha), \pi(\beta)) \notin E$, it is enough to check the following:

LEMma 19. If $\alpha(n) \neq \beta(n)$, then $\forall \gamma \in \Gamma_{n}(\gamma \cdot \pi(\alpha) \neq \pi(\beta))$.

Proof. Suppose, towards a contradiction, that there exists $\gamma \in \Gamma_{n}$ with $\gamma \cdot \pi(\alpha)=\pi(\beta)$. By reversing the roles of $\alpha$ and $\beta$ if necessary, we can assume that $\alpha(n)<\beta(n)$. Set $s=\alpha \mid n$ and $t=\beta \mid n$, and put

$$
x=\gamma_{n, \alpha(n)}^{-1} \gamma_{s}^{-1} \cdot \pi(\alpha), \quad y=\gamma_{n, \beta(n)}^{-1} \gamma_{t}^{-1} \cdot \pi(\beta),
$$

noting that these are both elements of $B_{n+1}$. As $\gamma \gamma_{s} \gamma_{n, \alpha(n)} \cdot x=\gamma_{t} \gamma_{n, \beta(n)} \cdot y$, it follows that $\gamma_{t}^{-1} \gamma \gamma_{s} \gamma_{n, \alpha(n)} \cdot x=\gamma_{n, \beta(n)} \cdot y$, thus

$$
\Delta_{n} \gamma_{n, \alpha(n)}\left(B_{n+1}\right) \cap \gamma_{n, \beta(n)}\left(B_{n+1}\right) \neq \emptyset,
$$

which contradicts condition (6).

It only remains to check that if $\alpha E_{\mathbf{k}} \beta$, then

$$
\varrho_{\mathbf{k}}(\alpha, \beta) /(1+\varepsilon) \leq \varrho(\pi(\alpha), \pi(\beta)) \leq \varrho_{\mathbf{k}}(\alpha, \beta)(1+\varepsilon) .
$$

Towards this end, suppose that $\alpha E_{\mathbf{k}} \beta$, fix $n \in \mathbb{N}$ such that $\forall m>n(\alpha(m)=$ $\beta(m))$, put $x=\pi(\alpha)$ and $y=\pi(\beta)$, and set $s=\alpha(0) \alpha(1) \ldots \alpha(n)$ and $t=\beta(0) \beta(1) \ldots \beta(n)$, noting that $\gamma_{s}^{-1} \cdot x=\gamma_{t}^{-1} \cdot y$, by Lemma 18. Put $\delta_{0}=1_{\Gamma}$, and for $i<n$, set $\delta_{i+1}=\gamma_{i, s(i)}^{-1} \delta_{i}$. Then

$$
\varrho\left(\gamma_{s}^{-1} \cdot x, x\right)=\varrho\left(\gamma_{n, s(n)}^{-1} \cdots \gamma_{0, s(0)}^{-1} \cdot x, x\right)=\prod_{i \leq n} \varrho\left(\gamma_{i, s(i)}^{-1} \delta_{i} \cdot x, \delta_{i} \cdot x\right),
$$

thus condition (4) ensures that

$$
\prod_{i \leq n} \varrho_{k_{i}}(0, s(i)) \leq \varrho\left(\gamma_{s}^{-1} \cdot x, x\right)<\prod_{i \leq n} \varrho_{k_{i}}(0, s(i))\left(1+\varepsilon_{i}\right) .
$$

An identical argument shows that

$$
\prod_{i \leq n} \varrho_{k_{i}}(0, t(i)) \leq \varrho\left(\gamma_{t}^{-1} \cdot y, y\right)<\prod_{i \leq n} \varrho_{k_{i}}(0, t(i))\left(1+\varepsilon_{i}\right)
$$


and since $\varrho(x, y)=\varrho\left(\gamma_{t}^{-1} \cdot y, y\right) / \varrho\left(\gamma_{s}^{-1} \cdot x, x\right)$, it follows that

$$
\begin{aligned}
\prod_{i \leq n} \varrho_{k_{i}}(0, t(i)) / \varrho_{k_{i}}(0, s(i))\left(1+\varepsilon_{i}\right) & \leq \varrho(x, y) \\
& \leq \prod_{i \leq n} \varrho_{k_{i}}(0, t(i))\left(1+\varepsilon_{i}\right) / \varrho_{k_{i}}(0, s(i)) .
\end{aligned}
$$

As $\prod_{i \leq n} \varrho_{k_{i}}(0, t(i)) / \varrho_{k_{i}}(0, s(i))=\prod_{i \leq n} \varrho_{k_{i}}(s(i), t(i))=\varrho_{\mathbf{k}}(\alpha, \beta)$, we obtain

$$
\varrho_{\mathbf{k}}(\alpha, \beta) / \prod_{i \leq n}\left(1+\varepsilon_{i}\right) \leq \varrho(x, y) \leq \varrho_{\mathbf{k}}(\alpha, \beta) \prod_{i \leq n}\left(1+\varepsilon_{i}\right)
$$

and $(\dagger)$ follows. This finishes the proof of Theorem 13.

We can now complete the proof of $(1) \Rightarrow(2)$ of Theorem 10 . Fix $\varepsilon>0$. By Theorem 13, there is a continuous $\varepsilon$-embedding $\pi: X_{\mathbf{k}} \rightarrow X$ of $\varrho_{\mathbf{k}}$ into $\varrho$, for some $\mathbf{k} \in \mathbb{N}^{\mathbb{N}}$ such that $\lim _{n \rightarrow \infty} k_{n}=\infty$. It follows from Lemma 12 that $\mu_{\mathbf{k}}$ is of type III, thus so too is the measure $\pi_{*} \mu_{\mathbf{k}}$ on $\pi\left(X_{\mathbf{k}}\right)$. As the cocycle $\pi_{*} \varrho_{\mathbf{k}} / \varrho \mid\left(E \mid \pi\left(X_{\mathbf{k}}\right)\right)$ is bounded, it follows from Proposition 9 that the cocycles $\pi_{*} \varrho_{\mathbf{k}}$ and $\varrho \mid\left(E \mid \pi\left(X_{\mathbf{k}}\right)\right)$ are cohomologous, thus Proposition 5 ensures that there is a $\varrho \mid\left(E \mid \pi\left(X_{\mathbf{k}}\right)\right)$-invariant, $\sigma$-finite measure $\mu \sim \pi_{*} \mu_{\mathbf{k}}$. By Theorem 1 of Feldman-Moore [2], there is a countable group of Borel automorphisms which generates $E$, and using this, we can easily extend $\mu$ to a $\varrho$-invariant, $\sigma$-finite measure on $X$ of type III.

With this final dichotomy result in hand, we can finally prove:

Theorem 20. Suppose that $X$ is a Polish space, $E$ is a countable Borel equivalence relation on $X$, and $\varrho: E \rightarrow(0, \infty)$ is a Borel cocycle. Then the following are equivalent:

(1) $\varrho$ is a coboundary.

(2) For every $\sigma$-finite measure $\mu$ on $X$, the following are equivalent:

(a) There is a $\sigma$-finite, E-invariant measure equivalent to $\mu$.

(b) There is a $\sigma$-finite, $\varrho$-invariant measure equivalent to $\mu$.

Proof. As Corollary 6 gives $(1) \Rightarrow(2)$, it is enough to show $(2) \Rightarrow(1)$. Towards this end, suppose that condition (2) holds, so that there are no $\varrho^{-}$ invariant, $\sigma$-finite measures of type III, which by Theorem 10 implies that $X \in \mathcal{I}_{\text {bounded }} \vee \mathcal{I}_{\text {discrete }}$. By Lemma 11 , there is an $E$-invariant Borel set $B \in \mathcal{I}_{\text {discrete }}$ such that $X \backslash B \in \mathcal{I}_{\text {bounded. }}$ Theorem 8 ensures that there are no atomless, $E \mid B$-ergodic, $\varrho \mid B$-invariant, $\sigma$-finite measures, and condition (2) then implies that there are no atomless, $E \mid B$-ergodic, $E \mid B$-invariant, $\sigma$-finite measures. It then follows from Theorem 7 that $B \in \mathcal{I}_{\text {smooth }}$, and since $\mathcal{I}_{\text {smooth }} \subseteq \mathcal{I}_{\text {bounded }}$, it follows that $X \in \mathcal{I}_{\text {bounded }}$, and Proposition 9 finally implies that $\varrho$ is a Borel coboundary. 
Acknowledgements. I would like to thank Mahendra Nadkarni, who first inspired me to explore the problem of coordinatewise decomposition. I would like to thank also Clinton Conley and the anonymous referee, who made stylistic suggestions and brought to my attention typos in earlier drafts of this paper.

\section{References}

[1] R. C. Cowsik, A. Kłopotowski, and M. G. Nadkarni, When is $f(x, y)=u(x)+v(y)$ ?, Proc. Indian Acad. Sci. Math. Sci. 109 (1999), 57-64.

[2] J. Feldman and C. Moore, Ergodic equivalence relations, cohomology, and von Neumann algebras. I, Trans. Amer. Math. Soc. 234 (1977), 289-324.

[3] A. Kechris, Classical Descriptive Set Theory, Grad. Texts in Math. 156, Springer, New York, 1995.

[4] B. Miller. On the existence of quasi-invariant measures of a given cocycle, preprint, 2004; available at http://www.math.ucla.edu/ ${ }^{b d m}$.

[5] S. Shelah and B. Weiss, Measurable recurrence and quasi-invariant measures, Israel J. Math. 43 (1982), 154-160.

Department of Mathematics

University of California

520 Portola Plaza

Los Angeles, CA, 90095-1555, U.S.A.

E-mail: bdm@math.ucla.edu

Received 9 December 2005;

in revised form 17 February 2006 